\title{
Bioinformatics analysis of mRNA and miRNA microarray to identify the key miRNA-gene pairs in small-cell lung cancer
}

\author{
YUN MAO $^{1,2^{*}}$, PENG XUE $^{2 *}$, LINLU LI $^{1,2}$, PENGPENG XU ${ }^{1,2}$, YAFANG CAI $^{1,2}$, \\ XUELEI CHU ${ }^{1,2}$, PENGYUAN JIANG ${ }^{1,2}$ and SHIJIE ZHU $^{2}$ \\ ${ }^{1}$ Graduate School, Beijing University of Chinese Medicine, Beijing 100029; ${ }^{2}$ Department of Oncology, \\ Wangjing Hospital, China Academy of Chinese Medical Sciences, Beijing 100102, P.R. China
}

Received January 22, 2019; Accepted June 6, 2019

DOI: $10.3892 / \mathrm{mmr} .2019 .10441$

\begin{abstract}
Small-cell lung cancer (SCLC) is a type of lung cancer with early metastasis, and high recurrence and mortality rates. The molecular mechanism is still unclear and further research is required. The aim of the present study was to examine the pathogenesis and potential molecular markers of SCLC by comparing the differential expression of mRNA and microRNA (miRNA) between SCLC tissue and normal lung tissue. A transcriptome sequencing dataset (GSE6044) and a non-coding RNA sequence dataset (GSE19945) were downloaded from the Gene Expression Omnibus (GEO) database. In total, 451 differentially expressed genes (DEGs) and 134 differentially expressed miRNAs (DEMs) were identified using the R limma software package and the GEO2R tool of the GEO, respectively. The Gene Ontology function was significantly enriched for 28 terms, and the Kyoto Encyclopedia of Genes and Genomes database had 19 enrichment pathways, mainly related to 'cell cycle', 'DNA replication' and 'oocyte meiosis mismatch repair'. The protein-protein interaction network was constructed using Cytoscape software to identify the molecular mechanisms of key signaling pathways and cellular activities in SCLC. The 1,402 miRNA-gene pairs encompassed 602 target genes of the DEMs using miRNAWalk, which is a bioinformatics platform that predicts DEM target genes and miRNA-gene pairs. There were 19 overlapping genes regulated by 32 miRNAs between target genes of the DEMs and DEGs. Bioinformatics analysis may help to better understand the role of DEGs, DEMs and miRNA-gene pairs in cell proliferation and signal transduction. The related hub
\end{abstract}

Correspondence to: Professor Shijie Zhu, Department of Oncology, Wangjing Hospital, China Academy of Chinese Medical Sciences, 6 South Central Road, Chaoyang, Beijing 100102, P.R. China

E-mail: 20180941234@bucm.edu.cn

*Contributed equally

Key words: small-cell lung cancer, bioinformatics analysis, mRNAs, microRNAs, microRNA-gene pairs genes may be used as biomarkers for the diagnosis and prognosis of SCLC, and as potential drug targets.

\section{Introduction}

Lung cancer is characterized by malignant neoplasms in the lung, with high morbidity and mortality rates worldwide (1). As a neuroendocrine tumor, small-cell lung cancer (SCLC) has the characteristics of strong invasiveness, and high recurrence and mortality rates, accounting for $\sim 13.6 \%$ of lung cancer (2). In the last few decades, there has been no significant improvement in the survival rate of patients with SCLC, and patients with SCLC have no obvious benefit from the current molecular targeted drugs (3). SCLC resistance to conventional treatment and its high recurrence rate are primarily due to its markedly high mutation rate and genomic instability (4). A previous study revealed that TP53 and RB transcriptional corepressor 1 (RB1) were the most frequently mutated genes in SCLC, with mutation frequencies of $\sim 85$ and $57 \%$, respectively (5). High prevalence of mutations in tumor suppressor genes TP53 and $\mathrm{RB} 1$, alterations in chromosome $3 \mathrm{p}$, has been revealed to be significantly correlated with a poor outcome (6). Mutations leading to dysregulated expression levels of a number of genes, including PIK3CA, PTEN, RPTOR independent companion or MTOR complex 2 and mTOR in the PI3K/AKT/mTOR pathway promote the cell cycle, inhibit apoptosis and facilitate early metastasis in SCLC (7). Gene amplifications of the MYC family, SOX2 and fibroblast growth factor receptor 1 were identified in patients with SCLC (8). Enhancer of zeste 2 polycomb repressive complex 2 subunit (EZH2) overexpression was associated with a higher degree of methylation of the EZH2 promoter, which had a considerable impact on SCLC cell viability (9).

MicroRNAs (miRNAs/miRs) are endogenous, highly conserved small RNAs, 20-24 nucleotides in length, which specifically bind to target mRNA to inhibit post-transcriptional gene expression. Mature miRNAs, in the miRNP riboprotein complex, are complementary to the 3 'untranslated region of the target gene to cleave or transfect the target gene mRNA $(10,11)$, and a single miRNA typically regulates dozens of genes. According to a previous study, $\sim 50 \%$ of the identified miRNAs are located on the genome at a tumor-associated fragile site, and are associated with tumor cell proliferation, differentiation 
and apoptosis (12). Therefore, miRNAs that are secreted by tumors can be used as biomarkers for different stages and different tumor types. The expression level of miR-25 in SCLC was revealed to be significantly upregulated and act as an oncogenic regulator by regulating cyclin E2 (13). miR-126 downregulation promoted overexpression of vascular endothelial growth factor A in lung cancer cell, thereby regulating angiogenesis (14). miR-17-92 and miR-1519-c directly interact with hypoxia-inducible factor 1- $\alpha$, affecting tumor angiogenesis (15). In the present study, bioinformatics tools were used to analyze the SCLC expression profile chips in a public gene chip database, which provided a theoretical basis for the biological functions of related genes and their molecular mechanisms that were involved in the occurrence and development of SCLC.

\section{Materials and methods}

Microarray data. Gene chip data were screened using the Gene Expression Omnibus (GEO; http://www.ncbi.nlm.nih.gov/geo) database, a public genomic database containing the entire gene expression data, chips and microarrays. Human SCLC sample gene expression profile public datasets GSE19945 (https://www.ncbi.nlm.nih.gov/geo/query/acc.cgi) and GSE6044 (16) were downloaded from GEO. The GSE19945 dataset, which is a miRNA dataset, contains 43 samples by surgical resection, including 35 SCLC samples and eight normal lung tissue samples. The expression of miRNA was detected using the Agilent Human 0.6K miRNA Microarray G4471A platform. The mRNA GSE6044 dataset, which was analyzed using the GPL201 [HG-Focus] Affymetrix Human HG-Focus Target Array platform, contained 14 samples by surgical resection, including nine SCLC samples and five normal lung tissue samples.

Differentially expressed genes (DEGs) and differentially expressed miRNAs (DEMs). The raw data of GSE6044 in the CEL file was effectively processed using the Affy package pair in R, using correction, normalization and $\log _{2}$ conversion (17). The DEGs in SCLC tissue compared with normal lung tissue were determined using limma package (18). DEGs were screened with a false discovery rate (FDR) corrected $\mathrm{P}<0.05$ and $\mid \log$ fold-change (FC) $\mid>1$. The DEMs in SCLC tissue compared with normal lung tissue were confirmed using the GEO2R application from GEO. The false FDR corrected $\mathrm{P}<0.05$ and $\mid \log \mathrm{FCl}>1$ were used as the screening thresholds.

Functional enrichment analysis of DEGs. DAVID (https://david.ncifcrf.gov/), a widely used web-based genomic functional annotation tool, was used for data annotation analysis (19). In the present study, DEGs were subjected to molecular function and pathway studies by Gene Ontology (GO) analysis and Kyoto Encyclopedia of Genes and Genomes (KEGG) pathway analysis.

Construction of protein-protein interaction (PPI) networks and module research. The PPI network of the DEGs was constructed using Cytoscape software (version 3.6.0; www. cytoscape.org) to identify the molecular mechanisms of key signaling pathways and cellular activities in SCLC. An interaction score $>0.4$ was considered to identify the significant PPIs.
Using the Network Analyzer plug-in of Cytoscape software, the association between the genes was analyzed, according to network topology characteristics such as the Clustering coefficient of the network, distribution of node degree and shortest path (20). Subsequently, molecular complex detection (MCODE) was used to confirm the hub genes. The screening thresholds were 'degree cutoff $=2$ ', 'node score cutoff $=0.2$ ', 'k-core $=2$ ' and 'max'depth $=100$ '.

miRNA target prediction. miRNAWalk is a bioinformatics platform for predicting DEM target genes and miRNA-gene pairs. In the present study, the targets of the DEMs were predicted using eight databases: miRWalk (21), miRanda (22), RNA22 (https://cm.jefferson.edu), miRDB (23), TargetScan (http://www.targetscan.org), miRMap (https://mirmap.ezlab. org), miRNAMap (http://mirnamap.mbc.nctu.edu.tw) and PITA (https:/genie.weizmann.ac.il). The screening criterion was that the miRNA target exists in the eight databases concurrently. The Venny 2.1 Online Tool (http://bioinfogp.cnb. csic.es) was used to find overlapping genes between DEGs and predictive genes of DEMs. The miRNA-gene negative regulatory network was depicted and visualized using Cytoscape software.

\section{Results}

DEGs and DEMs. The data was successfully normalized to ensure the accuracy of the data. In the GSE6044 dataset, 451 DEGs were identified in the SCLC samples compared with the normal lung tissue samples, of which 205 were downregulated and 246 were upregulated. In total, 20 DEGs with the lowest P-value are presented in Table I. Compared with normal lung tissue specimens, 134 DEMs were detected in SCLC specimens, of which 86 were downregulated and 49 were upregulated. The 20 DEMs with the lowest P-value are presented in Table II.

GO analysis of DEGs. The biological classification of the DEGs was analyzed utilizing the functional enrichment analysis of the DAVID website. The GO functional enrichment resulted in a total of 421 DEGs mapped to 403 GO terms. With the FDR corrected $\mathrm{P}<0.05$ as the significant enrichment criterion, 28 significant enriched functional clusters were screened (Fig. 1). In total, $46.43 \%$ (13) GO terms were significantly enriched in cellular components, mainly involving nuclear components, including 'nucleus', 'nucleoplasm', 'spindle', 'chromosome' and 'kinetochore'. Enrichment of $11 \mathrm{GO}$ terms, such as ' $\mathrm{G}_{1} / \mathrm{S}$ transition of mitotic cell cycle', 'cell division', 'mitotic nuclear division', 'DNA replication', 'mitotic sister chromatid segregation', 'sister chromatid cohesion', 'positive regulation of cell proliferation' and 'DNA replication initiation', belonged to biological processes. A total of four molecular functions were enriched, mainly involving binding-related terms, such as 'protein binding', 'calcium-dependent protein binding' and 'damaged DNA binding'.

Pathway analysis of DEGs. The present study used the DAVID online tool to perform KEGG enrichment analysis on 451 DEGs. A total of 227 DEGs were mapped into the KEGG database, and $\mathrm{P}<0.05$ was used as an enrichment screening standard. In total, 19 enriched functional clusters of the DEGs 
Table I. Top 20 DEGs between SCLC tissues compared with normal lung tissues.

\begin{tabular}{lcc}
\hline Gene name & Adjusted P-value & Log FC \\
\hline FABP6 & $1.77 \times 10^{-4}$ & -1.78 \\
IL17RB & $1.77 \times 10^{-4}$ & 1.49 \\
ACAA2 & $1.77 \times 10^{-4}$ & 2.12 \\
CDKN2A & $1.77 \times 10^{-4}$ & 2.33 \\
MARCKSL1 & $2.36 \times 10^{-4}$ & 2.10 \\
TMSB15 & $2.36 \times 10^{-4}$ & 4.6 \\
GSTA1 & $3.14 \times 10^{-4}$ & -4.26 \\
CYP4B1 & $3.14 \times 10^{-4}$ & -3.34 \\
TSPAN8 & $3.14 \times 10^{-4}$ & -2.94 \\
CES1 & $3.14 \times 10^{-4}$ & -2.51 \\
PDLIM4 & $3.14 \times 10^{-4}$ & -1.41 \\
CYP2J2 & $3.14 \times 10^{-4}$ & -1.13 \\
HDAC2 & $3.14 \times 10^{-4}$ & 1.39 \\
ID4 & $3.14 \times 10^{-4}$ & 1.99 \\
SOX4 & $3.14 \times 10^{-4}$ & 2.24 \\
MCM6 & $3.14 \times 10^{-4}$ & 2.32 \\
TOP2A & $3.14 \times 10^{-4}$ & 2.75 \\
TYMS & $3.14 \times 10^{-4}$ & 2.88 \\
MEST & $3.14 \times 10^{-4}$ & 3.78 \\
UCHL1 & $3.37 \times 10^{-4}$ & 3.08 \\
\hline
\end{tabular}

DEGs, differentially expressed genes; SCLC, small cell lung cancer; FC, fold-change.
Table II. Top 20 DEMs between SCLC tissues compared with normal lung tissues.

\begin{tabular}{lcr}
\hline miRNAs & Adjusted P-value & Log FC \\
\hline hsa-miR-96 & $1.31 \times 10^{-12}$ & 3.82 \\
hsa-miR-126 & $1.67 \times 10^{-12}$ & -3.77 \\
hsa-miR-183 & $7.50 \times 10^{-12}$ & 4.58 \\
hsa-miR-182 & $2.27 \times 10^{-11}$ & 3.72 \\
hsa-miR-638 & $3.72 \times 10^{-11}$ & -2.93 \\
hsa-miR-1 & $9.33 \times 10^{-11}$ & -4.64 \\
hsa-miR-130b & $1.85 \times 10^{-10}$ & 4.21 \\
hsa-miR-451 & $3.25 \times 10^{-9}$ & -4.22 \\
hsa-miR-144 & $5.12 \times 10^{-9}$ & -4.61 \\
hsa-miR-145 & $6.85 \times 10^{-9}$ & -3.88 \\
hsa-miR-26a & $7.48 \times 10^{-9}$ & -1.82 \\
hsa-miR-486-5p & $2.16 \times 10^{-8}$ & -4.04 \\
hsa-miR-301b & $2.16 \times 10^{-8}$ & 3.91 \\
hsa-miR-26b & $2.18 \times 10^{-8}$ & -1.92 \\
hsa-miR-338-3p & $2.66 \times 10^{-8}$ & -3.79 \\
hsa-miR-140-3p & $3.19 \times 10^{-8}$ & -2.40 \\
hsa-miR-140-5p & $3.25 \times 10^{-8}$ & -1.99 \\
hsa-miR-18a & $3.25 \times 10^{-8}$ & 3.35 \\
hsa-miR-498 & $4.75 \times 10^{-8}$ & -2.22 \\
hsa-miR-7 & $4.75 \times 10^{-8}$ & 5.08
\end{tabular}

DEMs, differentially expressed miRNAs; SCLC, small cell lung cancer; FC, fold-change.

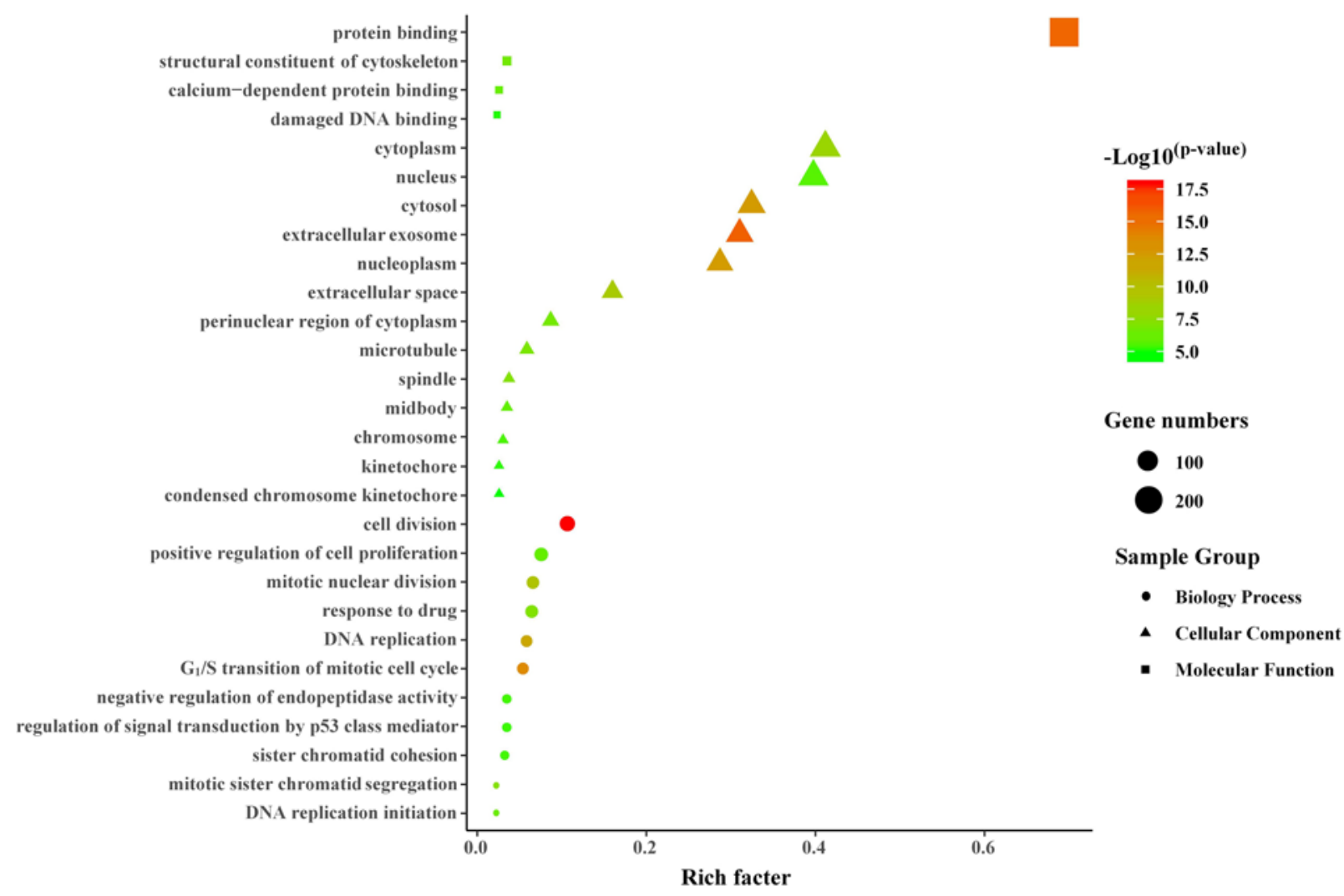

Figure 1. GO analysis of DEGs in the GSE6044 dataset. GO, Gene Ontology; DEGs, differentially expressed genes. 


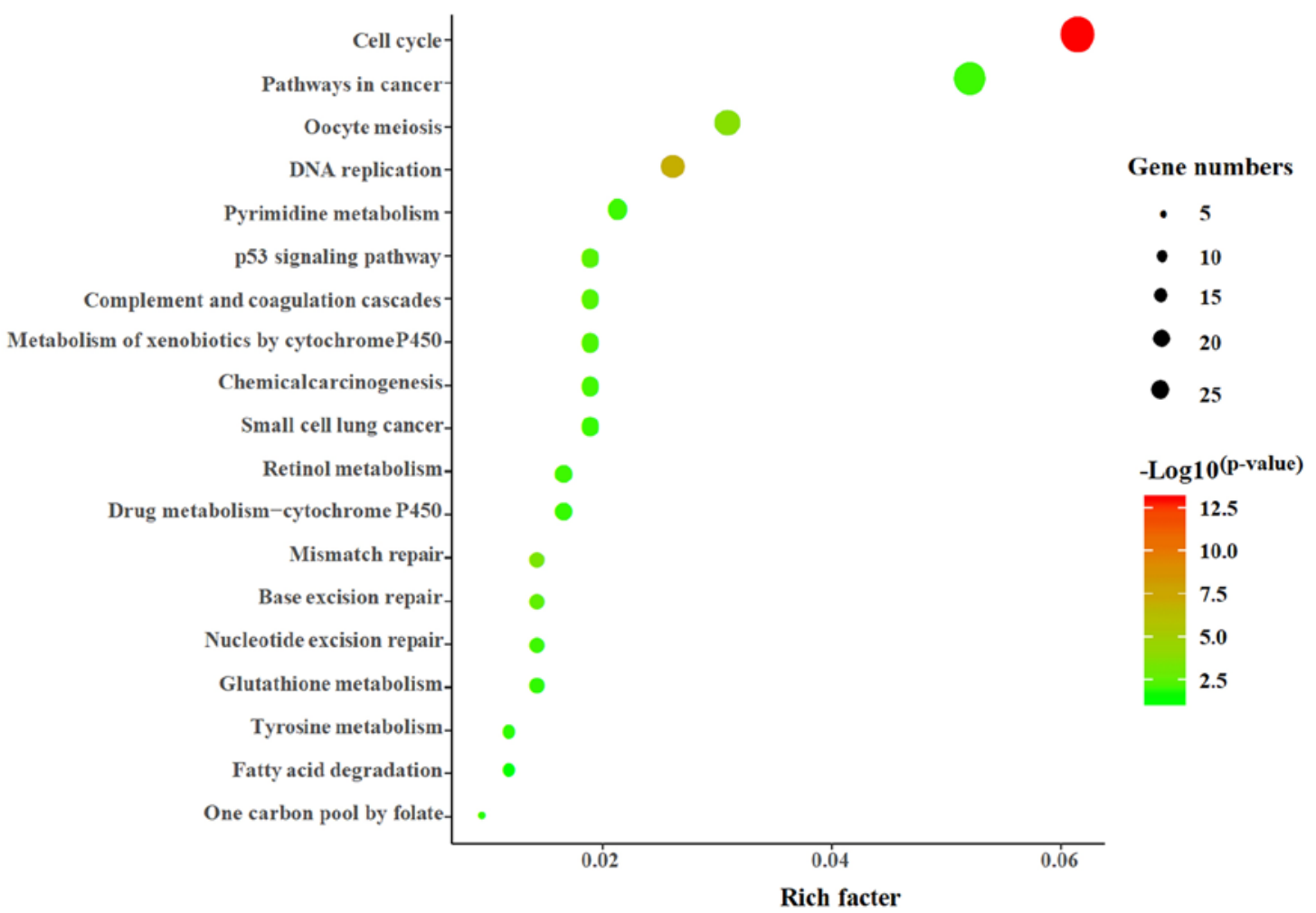

Figure 2. KEGG analysis of DEGs in the GSE6044 dataset. KEGG, Kyoto Encyclopedia of Genes and Genomes; DEGs, differentially expressed genes.

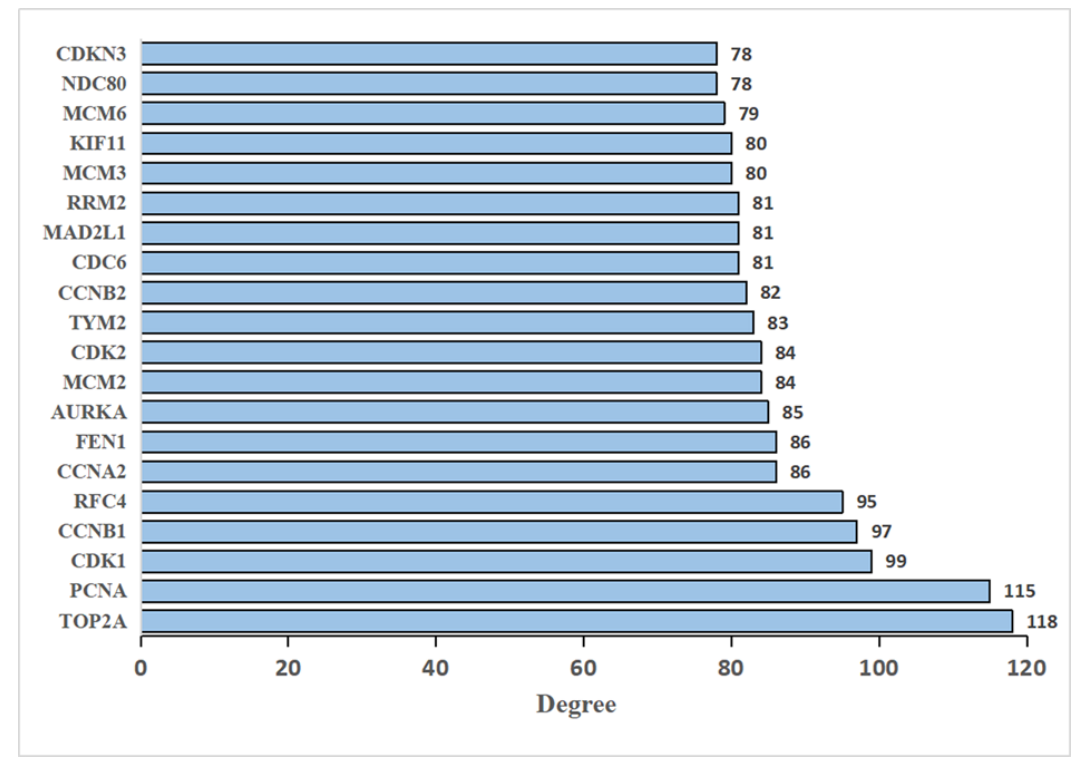

Figure 3. Top 20 hub genes in SCLC. SCLC, small cell lung cancer.

were obtained (Fig. 2), including 'cell cycle' (26 genes), 'pathways in cancer' ( 22 genes), 'oocyte meiosis' (13 genes), 'DNA replication' (11 genes), 'p53 signaling pathway', 'chemical carcinogenesis', 'drug metabolism-cytochrome p450' and 'mismatch repair'.

Construction of PPI networks. The visual PPI network of 451 DEGs was constructed using Cytoscape software. The isolated nodes and partially loosely connected gene nodes were removed, and the remaining DEGs together constituted a complex multi-center interaction network map to examine the association between DEGs, which contained 425 nodes and 3,770 edges. Among the 425 nodes, 10 DEGs with the highest degree of nodes were screened based on the Cytoscape software analysis results (Fig. 3). The results were as follows: DNA topoisomerase II $\alpha$ (TOP2A), proliferating cell nuclear antigen (PCNA), CDK1, cyclin B1 (CCNB1), replication factor C subunit 4 (RFC4), cyclin A2 (CCNA2), flap structure-specific 

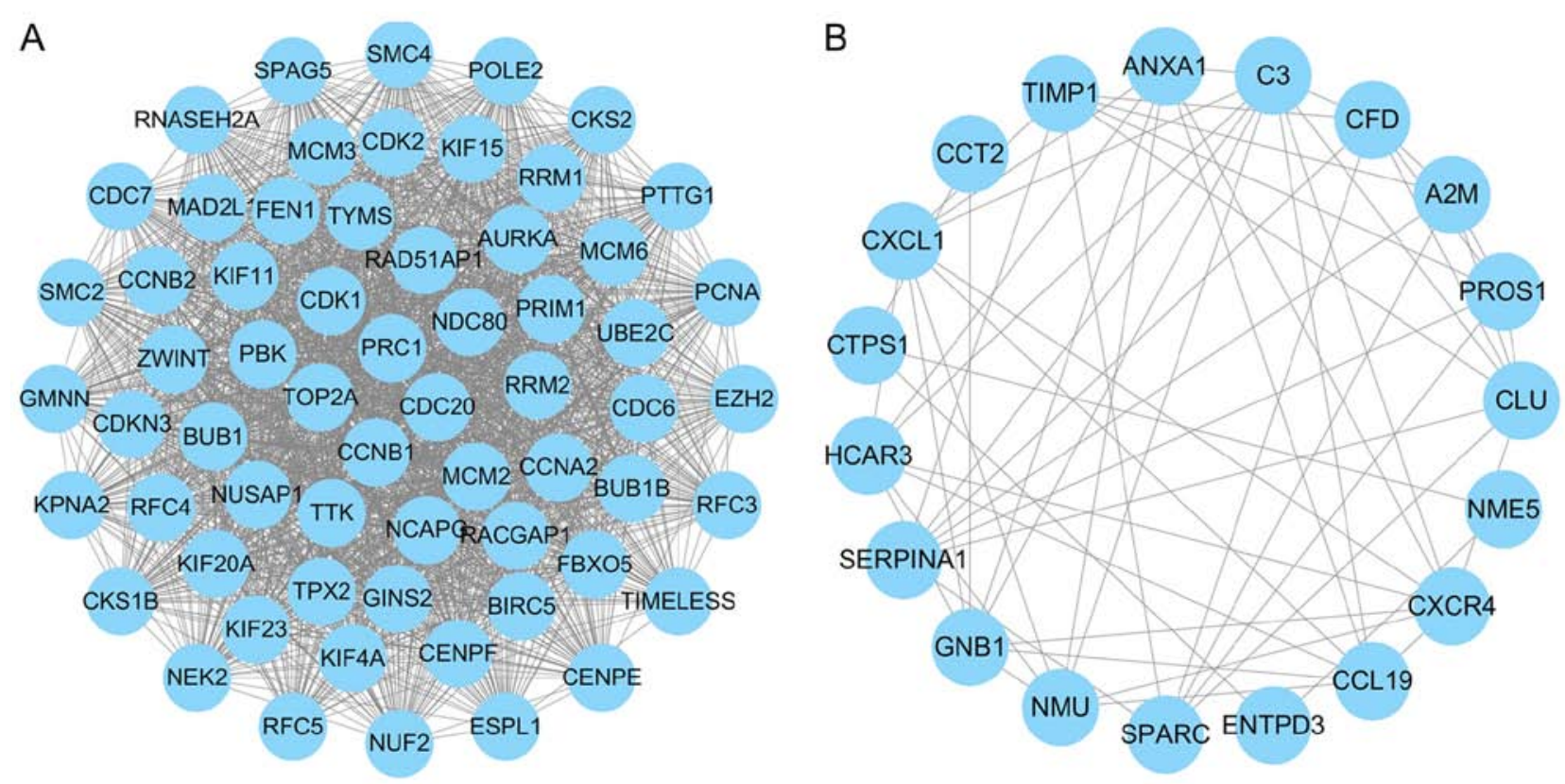

Figure 4. Top 2 modules from the protein-protein interaction network analysis. (A) Module 1 and (B) module 2.

Table III. KEGG analysis of module 1 and module 2.

\begin{tabular}{|c|c|c|c|c|}
\hline Category & Term & P-value & Count & Genes \\
\hline \multirow[t]{9}{*}{ Module 1} & hsa04110: Cell cycle & $2.48 \times 10^{-21}$ & 18 & $\begin{array}{l}\text { CDC7, CDC6, CDK1, TTK, CDC20, ESPL1, } \\
\text { PTTG1, MCM2, MCM3, CDK2, MCM6, } \\
\text { CCNB1, MAD2L1, CCNB2, BUB1, PCNA, } \\
\text { BUB1B, CCNA2 }\end{array}$ \\
\hline & hsa03030: DNA replication & $6.53 \times 10^{-16}$ & 11 & $\begin{array}{l}\text { RFC5, PRIM1, RFC3, RFC4, POLE2, PCNA, } \\
\text { MCM2, MCM3, RNASEH2A, FEN1, MCM6 }\end{array}$ \\
\hline & hsa04114: Oocyte meiosis & $1.04 \times 10^{-10}$ & 11 & $\begin{array}{l}\text { CCNB1, CDK1, MAD2L1, CCNB2, BUB1, } \\
\text { FBXO5, AURKA, CDC20, ESPL1, PTTG1, } \\
\text { CDK2 }\end{array}$ \\
\hline & $\begin{array}{l}\text { hsa04914: Progesterone-mediated oocyte } \\
\text { maturation }\end{array}$ & $4.16 \times 10^{-6}$ & 7 & $\begin{array}{l}\text { CCNB1, CDK1, MAD2L1, CCNB2, BUB1, } \\
\text { CCNA2, CDK2 }\end{array}$ \\
\hline & hsa03420: Nucleotide excision repair & $8.58 \times 10^{-5}$ & 5 & RFC5, RFC3, RFC4, POLE2, PCNA \\
\hline & hsa03430: Mismatch repair & $1.99 \times 10^{-4}$ & 4 & RFC5, RFC3, RFC4, PCNA \\
\hline & hsa04115: p53 signaling pathway & $3.43 \times 10^{-4}$ & 5 & CCNB1, CDK1, CCNB2, RRM2, CDK2 \\
\hline & hsa00240: Pyrimidine metabolism & $1.62 \times 10^{-3}$ & 5 & PRIM1, TYMS, POLE2, RRM2, RRM1 \\
\hline & hsa03410: Base excision repair & $1.20 \times 10^{-2}$ & 3 & POLE2, PCNA, FEN1 \\
\hline \multirow[t]{3}{*}{ Module 2} & $\begin{array}{l}\text { hsa04610: Complement and coagulation } \\
\text { cascades }\end{array}$ & $6.19 \times 10^{-4}$ & 5 & A2M, C3, SERPINA1, CFD, PROS1 \\
\hline & hsa04062: Chemokine signaling pathway & $4.55 \times 10^{-3}$ & 4 & CXCL1, CXCR4, GNB1, CCL19 \\
\hline & hsa00240: Pyrimidine metabolism & $1.50 \times 10^{-2}$ & 3 & NME5, CTPS1, ENTPD3 \\
\hline
\end{tabular}

$\mathrm{P}<0.05$ was used as an enrichment screening standard. 'Count' corresponds to the number of enriched genes in each term. KEGG, Kyoto Encyclopedia of Genes and Genomes.

endonuclease 1 (FEN1), aurora kinase A (AURKA), minichromosome maintenance complex component 2 (MCM2) and CDK2. The key modules were obtained using MCODE, with 95 hub genes with a genomic degree $\geq 5$. The two key modules with the highest degree were screened, and the functional and pathway enrichment of genes in these two modules was analyzed using DAVID online tools (Fig. 4A and B). Module 1 contained 62 nodes and 1,753 edges, mainly involved in 'cell cycle', 'DNA replication' and 'oocyte meiosis'. Module 2 was comprised of 19 nodes and 59 edges, which were associated with 'complement and coagulation cascades', 'chemokine signaling pathway' and 'pyrimidine metabolism' (Table III). 


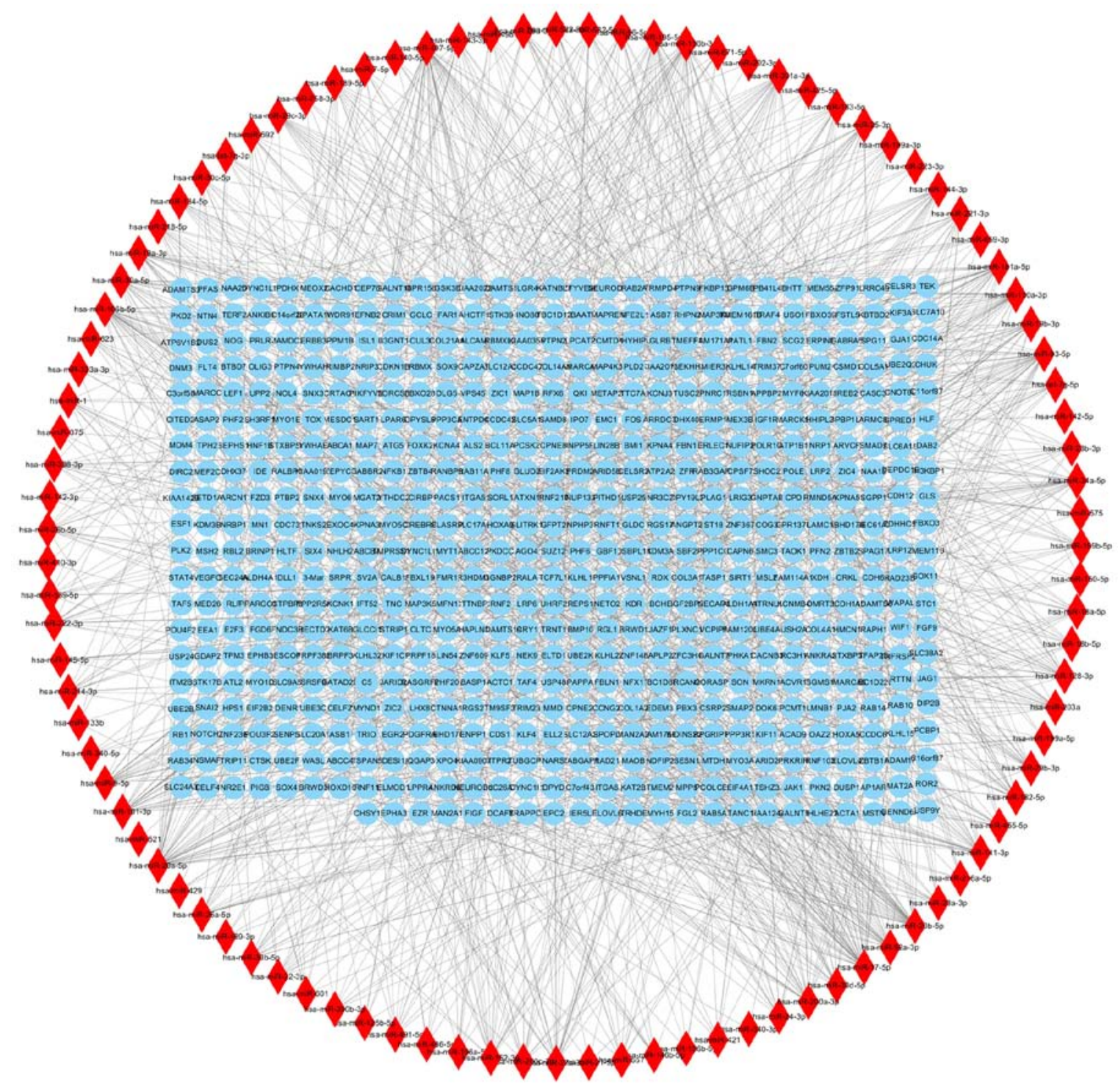

Figure 5. PPI network of miRNA-gene pairs. Diamonds represent miRNAs, and circles represent target genes predicted by miRNAs. PPI, protein-protein interaction; miRNAs, microRNAs.

miRNA-gene regulatory network. The miRNAWalk platform identified 1,402 miRNA-gene regulatory pairs containing 602 target genes of the DEMs (Fig. 5). There were 19 overlapping genes between target genes of the DEMs and DEGs. As presented in Fig. 6A, a total of 19 overlapping genes [lamin B1 (LMNB1), SOX11, fibroblast growth factor 9 (FGF9), Kruppel like factor 5 (KLF5), polypyrimidine tract binding protein 2 (PTBP2), collagen type IV $\alpha 1$ chain (COL4A1), nucleolar protein 4 (NOL4), cadherin EGF LAG seven-pass G-type receptor 3 (CELSR3), HLF transcription factor (HLF), PAR bZIP family member, kinesin family member 11 (KIF11), cysteine and glycine rich protein 2 (CSRP2), CDP-diacylglycerol synthase 1 (CDS1), aldehyde dehydrogenase 1 family member A1 (ALDH1A1), ISL LIM homebox 1 (ISL1), RAD21 cohesin complex component (RAD21), SOX4, erythrocyte membrane protein band 4.1 like 4B (EPB41L4B), fibulin 1 (FBLN1) and mutS homolog 2 (MSH2)] were regulated by 32 different miRNAs. The 19 significantly upregulated or downregulated genes and 32 DEMs are presented in a heat-map (Fig. 7A and B). In addition, three target genes (KIF11, MSH2 and RAD21) of the 19 overlapping genes belonged to the hub genes of GSE6044 and were regulated by five different miRNAs (miR-101, miR-21, miR-92a, miR-181a and miR-25; Figs. 6B and 8).

\section{Discussion}

SCLC is characterized by high malignancy and early extensive metastasis. The survival time of patients with extensive disease is only $8-13$ months and the 2 -year survival rate is $\sim 5 \%$ (24). Most patients are diagnosed with distant metastasis, which is often accompanied by poor prognosis (25), and the pathogenesis of SCLC at the molecular level is not clear. Therefore, there is an urgent need to identify more effective biomarkers for diagnosis and treatment. Microarray technology is useful in studying the changes in transcription and epigenetics of SCLC genes, which is an effective approach for identifying disease biomarkers. In addition, miRNAs affect the occurrence, metastasis and recurrence of SCLC by upregulating or downregulating gene expression levels. In the 


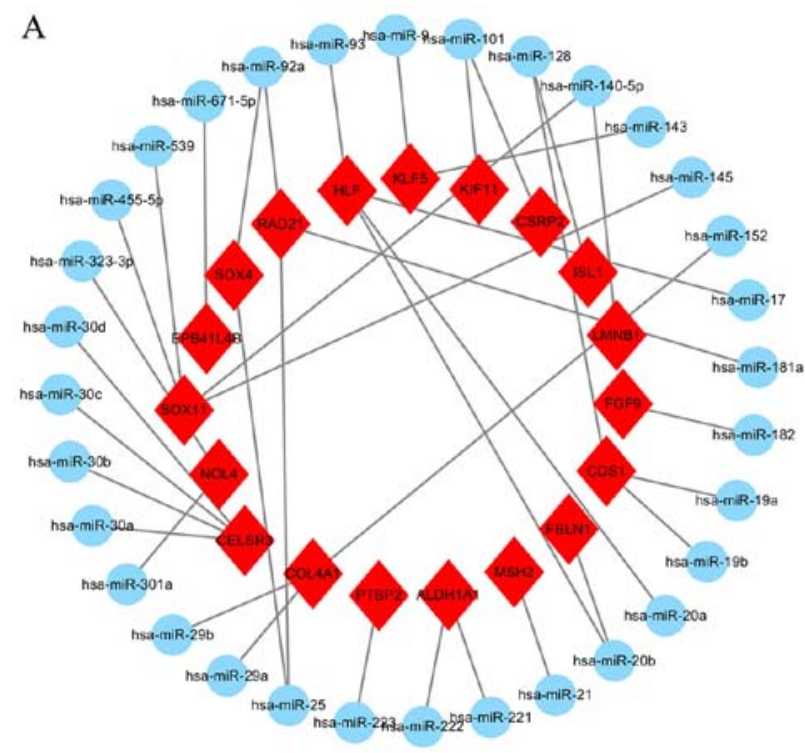

B

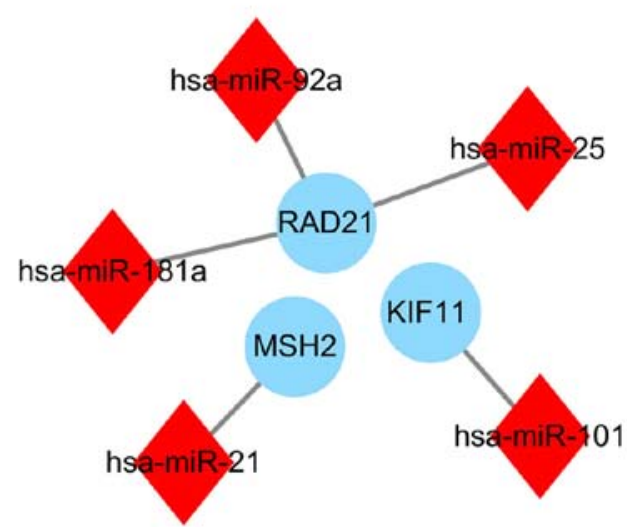

Figure 6. (A) PPI network of 19 target genes, which were regulated by 32 DEMs. (B) Three hub genes were regulated by 5 different miRNAs. PPI, protein-protein interaction; DEMs, differentially expressed miRNAs; miRNAs, microRNAs.

A

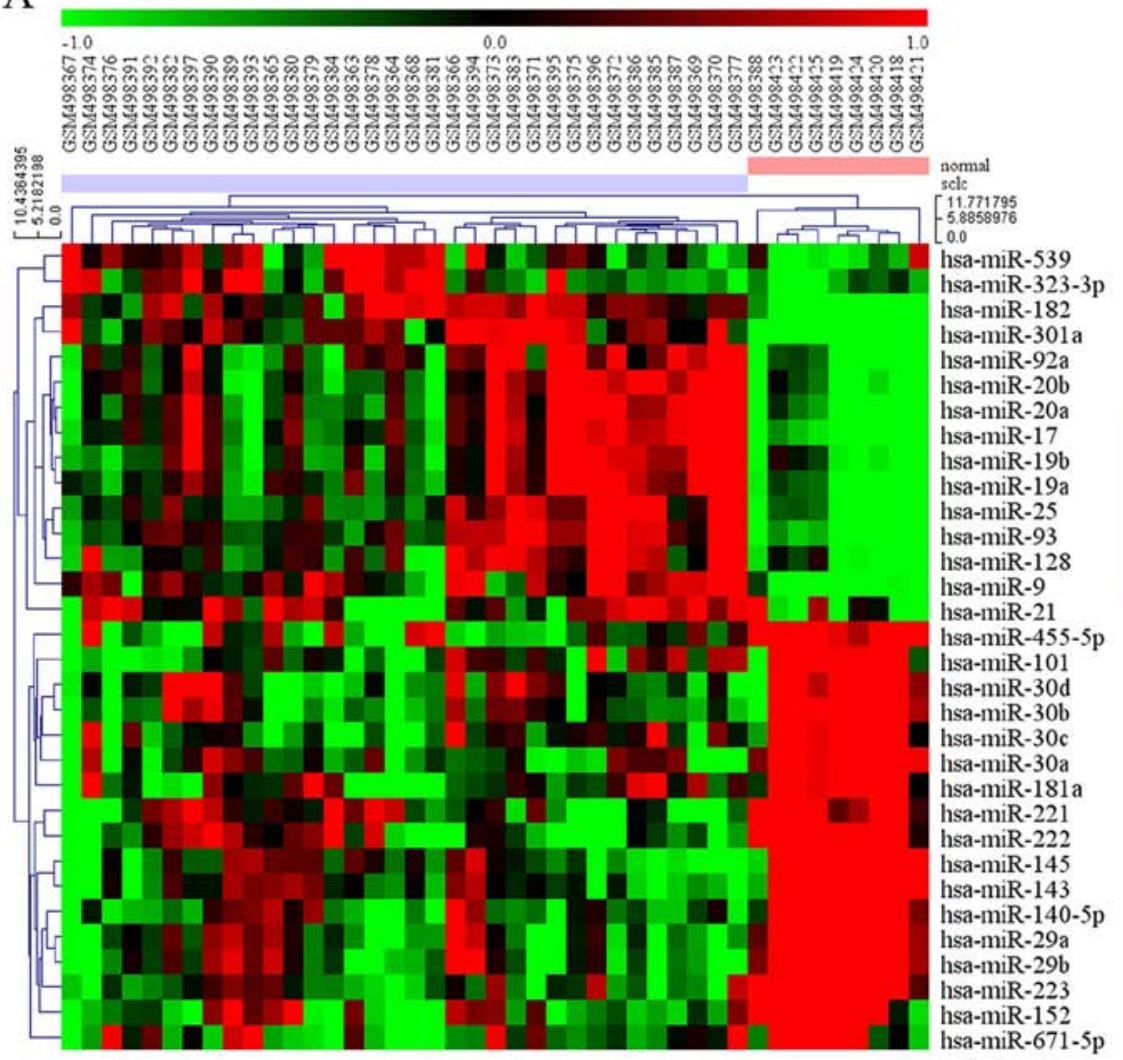

B

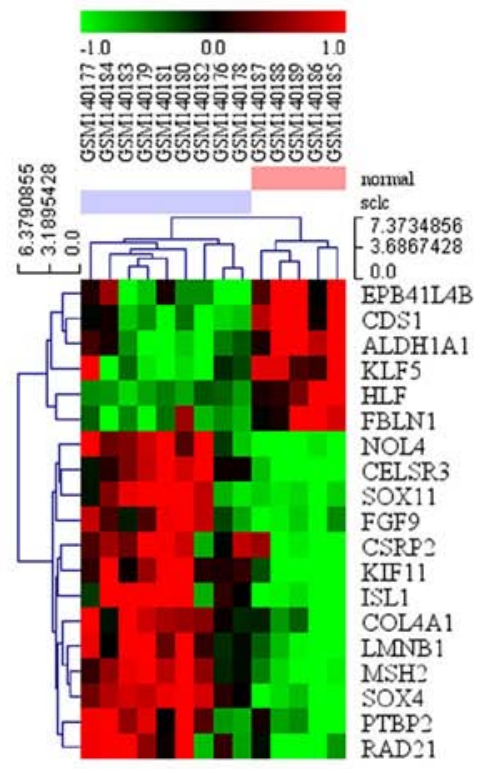

Figure 7. (A) Heat map revealing the expression profiles of 32 DEMs. (B) Heat map revealing the expression profiles of 19 DEGs. The colors depict high (red) and low (green) level of gene expression. The color alteration from green to black to red represents increasing expression. DEGs, differentially expressed genes; DEMs, differentially expressed miRNAs.

present study, bioinformatics technology was used to study the DEGs and DEMs of SCLC, and the miRNA-gene regulatory network was constructed using a software platform to examine the molecular pathological mechanisms of SCLC.

In the present study, 451 DEGs were identified from the GSE6044 dataset and subsequent bioinformatics analysis was conducted. The results of the KEGG and GO enrichment analysis of the DEGs revealed that the genes enriched in a number of signaling pathways, such as 'p53 signaling pathway', 'cell cycle', 'DNA replication', and 'oocyte meiosis' were mostly overexpressed, but the expression levels of genes aggregated in 'nucleotide excision repair', 'mismatch repair' and 'base 

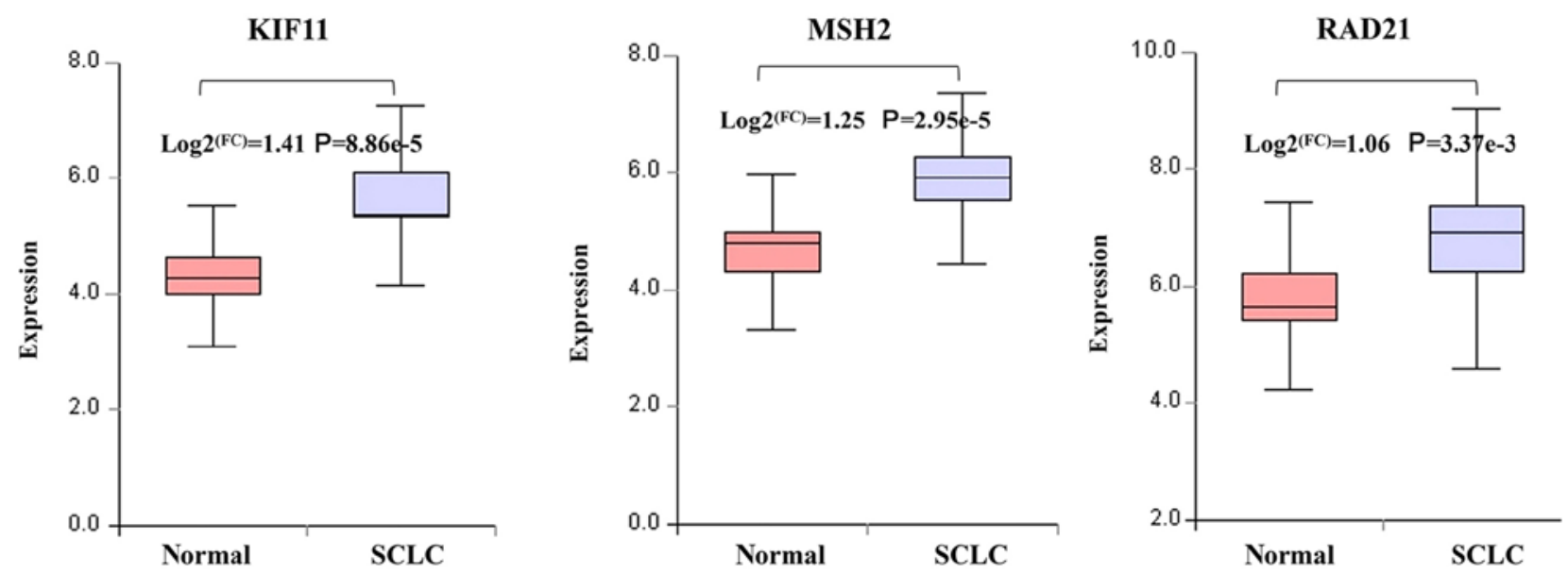

Figure 8. Box-plot revealing that the expression levels of KIF11, MSH2 and RAD21 are significantly different between SCLC tissues and normal lung tissues in the GSE6044 dataset. SCLC, small cell lung cancer; KIF11, kinesin family member 11; MSH2, mutS homolog 2; RAD21, RAD21 cohesin complex component.

excision repair' were mostly downregulated. p53 is a tumor suppressor gene that is activated during cell stress (including hypoxia, carcinogenesis and oxidative stress), inhibiting cell cycle progression and activating DNA repair mechanisms to promote cell survival and maintain genomic integrity (26). The aberrant expression of p53 in SCLC leads to the activation of related signaling pathways, accompanied by the activation of downstream genes, such as p21, MDM2, CKD1 and PCNA, which promotes proliferation and inhibits apoptosis of cancer cells (5). A significant marker of malignant tumors is the loss of control of the normal cell cycle. Tumor cells with a number of alterations lead to genomic instability and unscheduled proliferation (27). Notably, the loss or overexpression of kinases during cell mitosis leads to uncontrolled cell proliferation and carcinogenesis (28). Abnormal expression or methylation of key genes, including 8-oxoguanine DNA glycosylase, ERCC excision repair 3 , TFIIH core complex helicase subunit, AGT and ATM, in classical DNA repair pathways, such as nucleotide excision repair and mismatch repair, will result in loss of the ability to repair DNA damage caused by exogenous or endogenous gene toxicants, leading to tumorigenesis or drug resistance $(29,30)$. Furthermore, other enriched functional clusters contained complement and coagulation cascades, drug metabolism-cytochrome P450, glutathione metabolism and chemical carcinogenesis. The significance of complement cascade in malignant tumors has not been fully elucidated, however some researchers hypothesize that the complement cascade and its activation products can secrete growth factors required for survival of cancer cells $(8,31)$, and are also recruiters and inducers of immunosuppressive cells, which can produce reactive oxygen species, reactive nitrogen and other immunosuppressive molecules to help cancer cells escape the attack of the epidemic system (32). In addition, inhibiting the complement cascade and blocking programmed cell death 1 can delay the progression of lung cancer models (33). In the present study, 10 core network nodes were screened from the PPI network, which are considered the key genes for the occurrence and development of SCLC. For example, TOP2A is one of genes with the most nodes, and it is a subfamily of DNA topoisomerase II, which controls and changes the topological state of DNA during transcription. A previous study has shown that TOP2A is overexpressed in SCLC (34). The lower the differentiation degree of tumors, the higher the expression level of TOP2A (35).

In the present study, microarray analysis revealed that the occurrence mechanism of SCLC may be related to the expression of miRNA. Through the analysis of GSE19945 data, 86 were downregulated and 49 were upregulated, in which miR-1290 was the highest and miR-1 was the most significantly regulated. miR-1290 and miR-1 are abnormally expressed in a variety of malignancies; mir-1290 promoted the progression of non-small cell lung cancer by inhibiting MT1G (36), but there have only been a few studies on their role in SCLC. Subsequently, the integration analysis of the miRNA-gene regulatory pairs and DEGs revealed that there are 19 overlapping genes regulated by 32 different miRNAs. Further analysis revealed that three target genes (KIF11, MSH2 and RAD21) belong to the hub genes of GSE6044 and are regulated by five different miRNAs (miR-101, miR-21, miR-92a, miR-181a and miR-25). Among them, KIF11 (degree=75), MSH2 (degree=61) and RAD21 (degree=42) exhibited the highest degree, indicating that these three genes may play a core role in SCLC. The present study demonstrated that the decreased expression of miR-101 may lead to the upregulation of KIF11. As a tumor suppressor in malignant tumors, miR-101 has been revealed to be downregulated in various malignant tumors, such as lung, colon and breast cancer (37). A basic study revealed that miR-101 could directly inhibit the overexpression of EZH2 in lung cancer cells, and cooperate with paclitaxel to induce apoptosis, and inhibit invasion and cell proliferation (38). In addition, a meta-analysis of 2,088 tumor patients revealed that the low expression of miR-101 predicted poor overall survival and could be used as a predictive indicator for clinicopathological features (39). KIF11, also known as Eg5, is a homotetramer of BimC in kinesin family 5, whose overexpression leads to spindle defects and genetic instability, affecting cell division and proliferation, and is associated with tumor invasion, metastasis, recurrence and prognosis $(40,41)$.

In the present study, MSH2 was predicted to be a target of miR-21, which was upregulated in SCLC, according to the GSE6044 and GSE19945 datasets. The higher the malignant degree of tumors, the higher the expression level of miR-21, 
which is considered a potential marker for $\operatorname{SCLC}(42,43)$. miR-21 interacts with TLR (Toll-like receptor) family members to stimulate TLR-mediated pro-inflammatory responses leading to tumor growth and metastasis (44). Anti-miRNA-21-5p (AM21), consists of one tertiary amine, one quaternary amine and two tertiary lipids, and has been used to intervene in mouse lung cancer models. AM21 can prolong survival and slows tumor growth in mice with lung cancer (45). As an upstream factor, miR-21 can regulate the expression of MSH2. It was observed that MSH2 was highly expressed in SCLC, which was similar to a study by Fujii et al (46). Levallet et al (47) studied the expression of MSH2 in 681 cases of early lung cancer. The results revealed that high expression of MSH2 had poor prognosis and short survival time. The expression level of RAD21 is regulated by miR-92a, miR-181a and miR-25. RAD21 not only maintains sister chromatid binding and ensures correct replication, but also participates in DNA double strand break repair and meiotic recombination $(48,49)$. RAD21 is aberrantly expressed in various neoplasms such as breast, lung and rectal cancer, and serves a role in tumor development, prognosis and treatment (50-52). Similar to previous studies, the present results also indicated that miR-92a is overexpressed in SCLC. Highly expressed miR-92a enhances drug resistance and reduces survival of SCLC, and is thus considered a predictive biomarker for drug resistance and survival prognosis (53). miR-181a induces macrophage transformation into an M2 phenotype, which promotes macrophage-associated tumor cell metastasis by targeting KLF6 and C/EBP $\alpha$ (54). miR-25 has been revealed to be associated with cell proliferation and invasiveness in SCLC cell lines (13).

In conclusion, the present study analyzed gene and miRNA expression between lung cancer tissues and normal lung tissues using SCLC transcription sequencing data and non-coding RNA data from the GEO database, and identified aberrant expression of mRNAs and miRNAs in SCLC. Using bioinformatics analysis, the signaling pathways of aberrantly expressed gene enrichment were identified, and it was demonstrated that hub genes, such as KIF11, MSH2 and RAD21, are regulated by miRNAs. These genes are predicted to be biomarkers for the diagnosis, prognosis and therapeutic response prediction of SCLC. However, further research is required to verify the results.

\section{Acknowledgements}

Not applicable.

\section{Funding}

The present study was supported by the Natural Science Foundation of China (grant no. 81573915).

\section{Availability of data and materials}

All data generated or analyzed during this study are included in this published article.

\section{Authors' contributions}

YM, PX and SZ conceived and designed the present study, and wrote the manuscript. YM and PX contributed equally to this work. LL and PPX performed the data analysis. YC, XC and PJ collected the data. SZ reviewed the paper for intellectual content. All authors read and approved the final manuscript and agree to be accountable for all aspects of the work in ensuring that questions related to the accuracy or integrity of any part of the work are appropriately investigated and resolved.

\section{Ethics approval and consent to participate}

Not applicable.

\section{Patient consent for publication}

Not applicable.

\section{Competing interests}

The authors declare that they have no competing interests.

\section{References}

1. Bray F, Ferlay J, Soerjomataram I, Siegel RL, Torre LA and Jemal A: Global cancer statistics 2018: GLOBOCAN estimates of incidence and mortality worldwide for 36 cancers in 185 countries. CA Cancer J Clin 68: 394-424, 2018.

2. Xie D, Marks R, Zhang M, Jiang G, Jatoi A, Garces YI, Mansfield A, Molina $J$ and Yang P: Nomograms predict overall survival for patients with small-cell lung cancer incorporating pretreatment peripheral blood markers. J Thorac Oncol 10: 1213-1220, 2015.

3. Waqar SN and Morgensztern D: Treatment advances in small cell lung cancer (SCLC). Pharmacol Ther 180: 16-23, 2017.

4. Karachaliou N, Pilotto S, Lazzari C, Bria E, de Marinis F and Rosell R: Cellular and molecular biology of small cell lung cancer: An overview. Transl Lung Cancer Res 5: 2-15, 2016.

5. Sundaresan V, Lin VT, Liang F, Kaye FJ, Kawabata-Iwakawa R, Shiraishi K, Kohno T, Yokota J and Zhou L: Significantly mutated genes and regulatory pathways in SCLC-a meta-analysis. Cancer Genet 216-217: 20-28, 2017.

6. UdagawaH, Umemura S,Murakami I, Mimaki S, MakinoshimaH, Ishii G, Miyoshi T, Kirita K, Matsumoto S, Yoh K, et al: Genetic profiling-based prognostic prediction of patients with advanced small-cell lung cancer in large scale analysis. Lung Cancer 126: $182-188,2018$.

7. Sakre N, Wildey G, Behtaj M, Kresak A, Yang M, Fu P and Dowlati A: RICTOR amplification identifies a subgroup in small cell lung cancer and predicts response to drugs targeting mTOR. Oncotarget 8: 5992-6002, 2017.

8. Sharma SK, Chintala NK, Vadrevu SK, Patel J, Karbowniczek M and Markiewski MM: Pulmonary alveolar macrophages contribute to the premetastatic niche by suppressing antitumor T cell responses in the lungs. J Immunol 194: 5529-5538, 2015.

9. Poirier JT, Gardner EE, Connis N, Moreira AL, De Stanchina E, Hann CL and Rudin CM: DNA methylation in small cell lung cancer defines distinct disease subtypes and correlates with high expression of EZH2. Oncogene 34: 5869-5878, 2015.

10. Valencia-Sanchez MA, Liu J, Hannon GJ and Parker R: Control of translation and mRNA degradation by miRNAs and siRNAs. Genes Development 20: 515-524, 2006.

11. Catto JW, Antonio A, Bjartell AS, De Vere White R, Evans CP, Fussel S, Hamdy FC, Kallioniemi O, Mengual L, Schlomm T and Visakorpi T: MicroRNA in prostate, bladder, and kidney cancer: A systematic review. Eur Urol 59: 671-681, 2011.

12. Iqbal MA, Arora S, Prakasam G, Calin GA and Syed MA: MicroRNA in lung cancer: Role, mechanisms, pathways and therapeutic relevance. Mol Aspects Med, 2018 (Epub ahead of print).

13. Zhao Z, Liu J, Wang C, Wang Y, Jiang Y and Guo M: MicroRNA-25 regulates small cell lung cancer cell development and cell cycle through cyclin E2. Int J Clin Exp Pathol 7: 7726-7734, 2014.

14. Grimolizzi F, Monaco F, Leoni F, Bracci M, Staffolani S, Bersaglieri C, Gaetani S, Valentino M, Amati M, Rubini C, et al: Exosomal miR-126 as a circulating biomarker in non-small-cell lung cancer regulating cancer progression. Sci Rep 7: 15277, 2017. 
15. Grosso S, Doyen J, Parks SK, Bertero T, Paye A, Cardinaud B, Gounon P, Lacas-Gervais S, Noel A, Pouyssegur J, et al: MiR-210 promotes a hypoxic phenotype and increases radioresistance in human lung cancer cell lines. Cell Death Dis 4: e544, 2013.

16. Rohrbeck A, Neukirchen J, Rosskopf M, Pardillos GG, Geddert H, Schwalen A, Gabbert HE, von Haeseler A, Pitschke G, Schott M, et al: Gene expression profiling for molecular distinction and characterization of laser captured primary lung cancers. J Transl Med 6: 69, 2008.

17. Gautier L, Cope L, Bolstad BM and Irizarry RA: Affy-analysis of Affymetrix GeneChip data at the probe level. Bioinformatics 20 307-315, 2004

18. Diboun I, Wernisch L, Orengo CA and Koltzenburg M: Microarray analysis after RNA amplification can detect pronounced differences in gene expression using limma. BMC Genomics 7: 252, 2006

19. Dennis G Jr, Sherman BT, Hosack DA, Yang J, Gao W, Lane HC and Lempicki RA: DAVID: Database for annotation, visualization, and integrated discovery. Genome Biol 4: P3, 2003.

20. Assenov Y, Ramirez F, Schelhorn SE, Lengauer $T$ and Albrecht M: Computing topological parameters of biological networks. Bioinformatics 24: 282-284, 2008.

21. Dweep H, Sticht C, Pandey P and Gretz N: miRWalk-Database: Prediction of possible miRNA binding sites by 'walking' the genes of three genomes. J Biomed Inform 44: 839-847, 2011

22. Ma N and Gao X: $\beta$-Actin is predicted as one of the potential targets of miR-145: Choose internal control gene in verification of microRNA target. Carcinogenesis 34: 236, 2013.

23. Wang X: miRDB: A microRNA target prediction and functional annotation database with a wiki interface. RNA 14: 1012-1017, 2008.

24. Koinis F, Kotsakis A and Georgoulias V: Small cell lung cancer (SCLC): No treatment advances in recent years. Transl Lung Cancer Res 5: 39-50, 2016.

25. Travis WD: Update on small cell carcinoma and its differentiation from squamous cell carcinoma and other non-small cell carcinomas. Mod Pathol 25 (Suppl 1): S18-S30, 2012.

26. Issaeva N: p53 signaling in cancers. Cancers (Basel) 11 E332,2019.

27. Dominguez-Brauer C, Thu KL, Mason JM, Blaser H, Bray MR and Mak TW: Targeting mitosis in cancer: Emerging strategies. Mol Cell 60: 524-536, 2015.

28. Penna LS, Henriques JAP and Bonatto D: Anti-mitotic agents: Are they emerging molecules for cancer treatment? Pharmacol Ther 173: 67-82, 2017

29. Warkentin MT, Morris D, Bebb G and Brenner DR: The role of DNA repair capacity on lung cancer risk in never-smokers: A systematic review of epidemiologic studies. Cancer Treat Res Commun 13: 13-24,2017.

30. Jeggo PA, Pearl LH and Carr AM: DNA repair, genome stability and cancer: A historical perspective. Nat Rev Cancer 16: 35-42, 2016.

31. Reis ES, Mastellos DC, Ricklin D, Mantovani A and Lambris JD: Complement in cancer: Untangling an intricate relationship. Nat Rev Immunol 18: 5-18, 2018

32. Afshar-Kharghan V: The role of the complement system in cancer. J Clin Invest 127: 780-789, 2017.

33. Ajona D, Ortiz-Espinosa S, Moreno H, Lozano T, Pajares MJ, Agorreta J, Bertolo C, Lasarte JJ, Vicent S, Hoehlig K, et al: A combined PD-1/C5a blockade synergistically protects against lung cancer growth and metastasis. Cancer Discov 7: 694-703, 2017.

34. Wen P, Chidanguro T, Shi Z, Gu H, Wang N, Wang T, Li Y and Gao J: Identification of candidate biomarkers and pathways associated with SCLC by bioinformatics analysis. Mol Med Rep 18 $1538-1550,2018$

35. Neubauer E, Wirtz RM, Kaemmerer D, Athelogou M, Schmidt L, Sanger J and Lupp A: Comparative evaluation of three proliferation markers, Ki-67, TOP2A, and RacGAP1, in bronchopulmonary neuroendocrine neoplasms: Issues and prospects. Oncotarget 7: 41959-41973, 2016.

36. Zhang WC, Chin TM, Yang H, Nga ME, Lunny DP, Lim EK, Sun LL, Pang YH,Leow YN, Malusay SR, et al: Tumour-initiating cell-specific miR-1246 and miR-1290 expression converge to promote non-small cell lung cancer progression. Nat Commun 7 : 11702,2016

37. Kottakis F, Polytarchou C, Foltopoulou P, Sanidas I, Kampranis SC and Tsichlis PN: FGF-2 regulates cell proliferation, migration, and angiogenesis through an NDY1/KDM2B-miR-101-EZH2 pathway. Mol Cell 43: 285-298, 2011
38. Zhang JG, Guo JF, Liu DL, Liu Q and Wang JJ: MicroRNA-101 exerts tumor-suppressive functions in non-small cell lung cancer through directly targeting enhancer of zeste homolog 2 . J Thorac Oncol 6: 671-678, 2011.

39. Hu J, Wu C, Zhao X and Liu C: The prognostic value of decreased miR-101 in various cancers: A meta-analysis of 12 studies. Onco Targets Ther 10: 3709-3718, 2017.

40. Castillo A, Morse HC III, Godfrey VL, Naeem R and Justice MJ: Overexpression of Eg5 causes genomic instability and tumor formation in mice. Cancer Res 67: 10138-10147, 2007.

41. Karunagaran S, Subhashchandrabose S, Lee KW and Meganathan C: Investigation on the isoform selectivity of novel kinesin-like protein 1 (KIF11) inhibitor using chemical feature based pharmacophore, molecular docking, and quantum mechanical studies. Comput Biol Chem 61: 47-61, 2016.

42. Demes M, Aszyk C, Bartsch H, Schirren J and Fisseler-Eckhoff A Differential miRNA-expression as an adjunctive diagnostic tool in neuroendocrine tumors of the lung. Cancers (Basel) 8: E38, 2016.

43. Gao W, Xu J, Liu L, Shen H, Zeng H and Shu Y: A systematic-analysis of predicted miR-21 targets identifies a signature for lung cancer. Biomed Pharmacother 66: 21-28, 2012.

44. Fabbri M, Paone A, Calore F, Galli R, Gaudio E, Santhanam R, Lovat F, Fadda P, Mao C, Nuovo GJ, et al: MicroRNAs bind to Toll-like receptors to induce prometastatic inflammatory response. Proc Natl Acad Sci USA 109: E2110-E2116, 2012.

45. Florczuk M, Szpechcinski A and Chorostowska-Wynimko J: miRNAs as biomarkers and therapeutic targets in non-small cell lung cancer: Current perspectives. Targeted Oncol 12: 179-200, 2017.

46. Fujii K, Miyata Y, Takahashi I, Koizumi H, Saji H, Hoshikawa M, Takagi M, Nishimura T and Nakamura H: Differential proteomic analysis between small cell lung carcinoma (SCLC) and pulmonary carcinoid tumors reveals molecular signatures for malignancy in lung cancer. Proteomics Clin Appl 12: e1800015, 2018.

47. Levallet G, Dubois F, Fouret P, Antoine M, Brosseau S, Bergot E, Beau-Faller M, Gounant V, Brambilla E, Debieuvre D, et al: MSH2/BRCA1 expression as a DNA-repair signature predicting survival in early-stage lung cancer patients from the IFCT-0002 Phase 3 trial. Oncotarget 8: 4313-4329, 2017.

48. Bauerschmidt C, Arrichiello C,Burdak-Rothkamm S, Woodcock M, Hill MA, Stevens DL and Rothkamm K: Cohesin promotes the repair of ionizing radiation-induced DNA double-strand breaks in replicated chromatin. Nucleic Acids Res 38: 477-487, 2010.

49. Urban E, Nagarkar-Jaiswal S, Lehner CF and Heidmann SK: The cohesin subunit $\operatorname{Rad} 21$ is required for synaptonemal complex maintenance, but not sister chromatid cohesion, during Drosophila female meiosis. PLoS Genet 10: e1004540, 2014.

50. Xu H, Yan Y, Deb S, Rangasamy D, Germann M, Malaterre J, Eder NC, Ward RL, Hawkins NJ, Tothill RW, et al: Cohesin $\operatorname{Rad} 21$ mediates loss of heterozygosity and is upregulated via wnt promoting transcriptional dysregulation in gastrointestinal tumors. Cell Rep 9: 1781-1797, 2014.

51. Yan M, Xu H, Waddell N, Shield-Artin K, Haviv I, kConFab authors, McKay MJ and Fox SB: Enhanced RAD21 cohesin expression confers poor prognosis in BRCA2 and BRCAX, but not BRCA1 familial breast cancers. Breast Cancer Res 14: R69, 2012.

52. Rhodes DR, Yu J, Shanker K, Deshpande N, Varambally R, Ghosh D, Barrette T, Pandey A and Chinnaiyan AM: Large-scale meta-analysis of cancer microarray data identifies common transcriptional profiles of neoplastic transformation and progression. Proc Natl Acad Sci USA 101: 9309-9314, 2004

53. Ranade AR, Cherba D, Sridhar S, Richardson P, Webb C, Paripati A, Bowles B and Weiss GJ: MicroRNA 92a-2*: A biomarker predictive for chemoresistance and prognostic for survival in patients with small cell lung cancer. J Thorac Oncol 5: 1273-1278, 2010.

54. Bi J, Zeng X, Zhao L, Wei Q, Yu L, Wang X, Yu Z, Cao Y, Shan $F$ and Wei M: miR-181a induces macrophage polarized to M2 phenotype and promotes M2 macrophage-mediated tumor cell metastasis by targeting KLF6 and C/EBPalpha. Mol Ther Nucleic Acids 5: e368, 2016.

This work is licensed under a Creative Commons Attribution-NonCommercial-NoDerivatives 4.0 International (CC BY-NC-ND 4.0) License. 